\title{
Sensitivity Pattern of Gram Negative Bacteria Contaminating Traumatic Wounds: A Retrospective Study in a Tertiary Care Hospital of Kathamandu Valley
}

Satya Narayan Suwal', Sammodavardhana Kaundinnyayana ${ }^{2}$, Krishna Govinda Prajapati ${ }^{3}$ and $^{2}$

\section{Reena Baidya ${ }^{4}$}

${ }^{1}$ Department of General Practice and Emergency Medicine, Nepalese Army Institute of Health Sciences, Shree Birendra Hospital, Chhauni, Kathmandu, Nepal

${ }^{2}$ Department of Pharmacology, Nepalese Army Institute of Health Sciences, Syanobharyang, Kathmandu, Nepal

${ }^{3}$ Department of Microbiology, B and B Hospital, Gwarko, Lalitpur, Nepal

${ }^{4}$ Department of Pathology, B and B Hospital, Gwarko, Lalitpur, Nepal

\begin{abstract}
Introduction: Gram negative bacteria are commonly involved in wound infections and are significantly associated with morbidity and mortality because of increasing resistance to commonly used antibacterial agents. This study aims to evaluate the pattern of antibacterial sensitivity of the gram negative bacteria from wound swabs of accidental trauma cases.

Methods: This retrospective study was done by collecting all the culture positive reports for wound swabs of accidental wounds from the microbiology laboratory and analyzing the reports showing growth of gram negative bacteria for frequency of sensitivity to commonly used antibacterial agents.

Results: Culture positivity rate was $21.95 \%$ with growth of gram negative bacteria in $79.7 \%$ of cases. The most commonly isolated organism was Escherichia coli being $41.3 \%$ among gram negative isolates. Highly resistant bacteria were E. coli, Klebsiella pneumoniae and Acinetobacter spp.

Conclusions: As there is low culture positivity rate for wound swabs obtained before washing the wounds inflicted by accidental trauma, prolonged routine use of prophylactic antibiotic is not justified. Thoroughly washing the wound is very likely to decrease the rate of wound infection further and reduces the need of antibacterial agents. Culture and sensitivity test should guide the treatment when wound is infected because highly resistant gram negative organism may be involved in such cases.

Key words: colistin; culture and sensitivity; gram negative bacteria; multi-drug resistance
\end{abstract}

Correspondence: Satya Narayan Suwal, Department of General Practice and Emergency Medicine, Nepalese Army Institute of Health Sciences, Shree Birendra Hospital, Chhauni, Kathmandu, Nepal. E-mail: suwalsuwa12000@gmail.com

DOI: http://dx.doi.org/10.3126/mjsbh.v19i2.27689

Submitted on: 2020-02-13

Accepted on: 2020-04-14

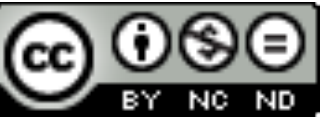

This work is licensed under creative common license:

http://creativecommons.org/licenses/by-nc-nd/4.0/ (C) MJSBH 2020 


\section{INTRODUCTION}

Wound infections continue to represent a major medical problem, both in terms of how they affect the outcome of surgical procedures (surgical site infections), and for their impact on the length of hospital stay and medical costs. ${ }^{1}$ Gram negative wound infections are important cause of morbidity and mortality in case of accidental wound infections such as road traffic accidents which are contaminated wounds. ${ }^{2}$ Emerging resistance especially multidrug resistance among aerobic Gram negative bacilli e.g. Acinetobacter spp., Pseudomonas spp., Klebsiella spp. is a big challenge and remains as one of the most important factor resulting in poor outcome of patients with wound infection. Gram negative infections may lead to sepsis with relatively high mortality as they are more commonly associated with septic shock and multi-organ failure. High degree of resistance in gram negative bacteria is a global problem and a study done in a tertiary care hospital in Kathmandu too has confirmed this in health care associated infections as well as pyogenic wound infections. ${ }^{3,4}$ Early recognition and appropriate antimicrobial treatment is very essential to prevent such complications and mortality. Though new modalities of treatment e.g. dressing with honey, have been found to be promising in management of wound infections treatment of more deeper infection and prevention of sepsis is still challenging. ${ }^{5}$

This study aims to find sensitivity pattern of gram negative bacteria isolated from accidental wounds in a tertiary care hospital in Lalitpur, Nepal. This may be helpful not only in understanding the evolution of antibacterial resistance in our country but also in developing guidelines for empirical treatment of wound infections based on local susceptibility profiles and developing strategies for containment of antimicrobial resistance that includes cycling the antimicrobial agents to preserve sensitivity. ${ }^{6}$

\section{METHODS}

This retrospective study was conducted from the records of culture and sensitivity reports for bacterial isolates in wound swab that arrived at the Microbiology laboratory from the patients visiting the emergency department of a tertiary care
Hospital of Lalitpur, Nepal because of accidental trauma resulting in apparent wound infection. Ethical approval from the Hospital IRC for the study was obtained in February 2020. The culture positive reports with growth of gram negative bacteria from July 2014 to July 2015 were collected and analysed descriptively for frequency of sensitivity and resistance of the gram negative bacteria using Microsoft Excel (Microsoft Office 2016)

Wound swabs were collected, before wound washing, with the help of sterile disposable cotton swabs and immediately inoculated onto blood agar and MacConkey agar media and incubated at $37^{\circ} \mathrm{C}$ for 24 hours. If there was no growth in 24 hours, the plate was observed at 48 hours for bacterial growth. Identification of bacteria from positive cultures was done with standard microbiological techniques which included Gram staining and biochemical reactions. The antibiotic sensitivity test of all isolates was performed by modified Kirby Bauer's disc diffusion method on Mueller Hinton agar using antibiotic discs of gentamicin (10 $\mu \mathrm{g})$, amikacin $(30 \mu \mathrm{g})$, ceftriaxone $(30 \mu \mathrm{g})$, cefoperazone with sulbactam $(75 / 30 \mu \mathrm{g})$, imipenem $(10 \mu \mathrm{g})$, meropenem $(10 \mu \mathrm{g})$, piperacillin with tazobactam $(100 / 10 \mu \mathrm{g})$, colistin sulfate $(10$ $\mu \mathrm{g})$, ofloxacin $(5 \mu \mathrm{g})$, ciprofloxacin $(5 \mu \mathrm{g}$, chloramphenicol $(30 \mu \mathrm{g})$ and ampicillin $(10 \mu \mathrm{g})$ as far as applicable to the isolated gram negative organism. Ceftazidime $(30 \mu \mathrm{g})$ was used in place of ceftriaxone for Pseudomonas aeruginosa (Hi Media Laboratories P. Ltd. Mumbai, India). Interpretations of antibiotic susceptibility results were made according to the guidelines of interpretative zone diameters of CLSI except for colistin. ${ }^{7}$ Escherichia coli ATCC 25922 and Pseudomonas aeruginosa ATCC 27853 were used as the control organisms for antibiotic sensitivity.

\section{RESULTS}

Culture positivity rate was $21.95 \%$ ( $79 / 360)$. Rate of infection with gram negative bacteria was $79.7 \%$ (63 / 79). The most commonly isolated organism was Escherichia coli, which was $41.3 \%$ among gram negative isolates. Other gram negative bacteria isolated were Acinetobacter spp, Klebsilella pneumoniae, Entetrobacter spp, Pseudomonas aeruginosa, Proteus vulgaris, 
Table 1. Sensitivity of gram negative isolates (in percentage)

\begin{tabular}{|c|c|c|c|c|c|c|}
\hline Antibacterial agents & $\begin{array}{c}\text { E.coli } \\
(n=26)\end{array}$ & $\begin{array}{l}\text { Acinetobacter } \\
\text { spp. }(n=11)\end{array}$ & $\begin{array}{c}\text { Klebsilella } \\
\text { pneumoniae } \\
\qquad(\mathrm{n}=9)\end{array}$ & $\begin{array}{l}\text { Entetrobacter } \\
\text { spp. }(n=9)\end{array}$ & $\begin{array}{l}\text { Pseudomonas } \\
\text { aeruginosa } \\
\qquad(\mathrm{n}=4)\end{array}$ & $\begin{array}{l}\text { Others } \\
(n=4)\end{array}$ \\
\hline Gentamicin & 84.6 & 63.6 & 66.7 & 88.9 & 100 & 50 \\
\hline Amikacin & 92 & 63.6 & 66.7 & 88.9 & 100 & 50 \\
\hline Piperacillin + tazobactam & 73 & 45.5 & 55.6 & 66.7 & 75 & 75 \\
\hline Ceftriaxone & 80.8 & 27.3 & 44.5 & $88.9 *$ & & 25 \\
\hline Cefoperazone + sulbactam & 80.8 & 63.6 & 55.6 & 55.6 & 50 & 50 \\
\hline Ceftazidime & - & - & - & - & 50 & - \\
\hline Meropenem & 80.8 & 45.5 & 77.8 & 88.9 & 100 & 75 \\
\hline Imipenem & 61.5 & 54.6 & 66.7 & 55.6 & 75 & 50 \\
\hline Ofloxacin & 80.8 & 54.6 & 66.7 & 88.9 & 100 & 75 \\
\hline Ciproploxacin & 76.9 & 54.6 & 66.7 & 88.9 & 100 & 75 \\
\hline Chloramphenicol & - & - & 33.3 & 88.9 & $*$ & 50 \\
\hline Colistin & 96.2 & 100 & 100 & 100 & 100 & 100 \\
\hline Ampicillin (Amoxicillin) & 7.7 & - & * & $*$ & $*$ & * \\
\hline
\end{tabular}

- = Not tested, $*=$ Intrinsicallv resistant

Proteus mirabilis, Enterobacter cloacae, Klebsiella oxytoca (One isolate each for last four organisms included as others in the table). Sensitivity rate of the gram negative isolates for different antibacterial agents is presented in Table 1 . Highly resistant $E$. coli, Klebsiella pneumoniae and Acinetobacter spp., two cases for each, sensitive only to colistin sulfate were isolated during the study period. There was one isolate of $E$. coli resistant to colistin sulfate but sensitive to all other tested agents except ampicillin. Emergence of multidrug-resistant (MDR) E. coli has been reported from different parts of the world and it is taken as urgent global health threat due to the lack of effective antimicrobial agents in recent decades. ${ }^{8}$ Resistance to imipenem in Enterobacter species is $44.4 \%$ which has not been reported by other studies done in Nepal. ${ }^{4,9-12}$

\section{DISCUSSION}

Although study on the postoperative wound infection done in another tertiary care hospital in Kathmandu has shown the rate of Gram positive infections to be higher (57.4\%) than Gram negative infections, this study shows the rate of Gram negative infections to be higher. ${ }^{4}$ This can be expected as organism involved in post-traumatic wound infection and surgical wound infections differ. The rate of gram negative infections is shown to be higher in a study done in India on wound infections in road traffic accident patients. ${ }^{12}$
Colistin, an old antibacterial, largely abandoned for systemic use because of systemic toxicity, has been reintroduced in this decade to treat serious multidrug resistant gram negative infections especially those with Pseudomonas and Acinetobacter species which are resistant to other classes of antibacterial agents. ${ }^{12}$ Klebsiella pneumoniae sensitive to colistin only (with MIC for imipenem $32 \mu \mathrm{g} / \mathrm{ml}$ ) had been isolated from central venous catheter in a case report of 2002 from Greece in a patient with multiple injuries from road traffic accident. ${ }^{15}$

Because colistin resistance has already been reported in some parts of the world, it may be expected in this region too though not reported yet. ${ }^{4,9-12,15}$ Combination of colistin with meropenem is shown ineffective in preventing emergence of resistance to colistin in a study. So judicious use of this valuable antibiotic seems to be the way to preserve sensitivity of the highly resistant organisms. ${ }^{16}$

Beta lactamases are the hydrolytic enzymes that destroy the functional part of $\beta$-lactam antibiotics used for the treatment of most of the Gram negative as well as Gram positive bacterial infections. Alarmingly increasing resistance in Gram negative isolates towards the extended spectrum penicillins, cephalosporins and carbapenems could be attributed to ESBL, Amp C $\beta$-lactamase, 
carbapenamase producers and some other relevant underlying mechanisms. ${ }^{17}$

Among the orally effective agents, ofloxacin and ciprofloxacin (second generation fluoroquinolones) were tested as most of the Gram negative bacteria are expected to be sensitive to them. The rate of sensitivity to ofloxacin as well as ciprofloxacin was comparable to meropenem for most of the isolated gram negative bacteria and better than imipenem for E. coli and Enterobacter spp. In addition, Pseudomanas aeruginosa isolates which were resistant to ceftazidime (Two out of four isolates) were still sensitive to both of the tested fluoroquinolones. Though the number of isolates is small and better conclusions can be drawn from large scale studies, reserving the fluoroquinolones may be prudent for maintaining the sensitivity of these bacteria towards this valuable group of antibiotics.

\section{CONCLUSIONS}

Gram negative bacilli are commonly involved in post-traumatic wound infections and they frequently show multi-drug resistance. Use of appropriate antibiotics for prophylaxis should be guided by local sensitivity data and treatment of such infections should be guided by wound swab culture and sensitivity report as far as possible. Routine prolonged course of single or multiple antibacterial agents for prophylaxis of wound infection is not justified because the rate of culture positivity is low even with the swabs taken before washing the wounds as shown by the study. Reservation of fluoroquinolones for treatment of serious Gram negative infection may be advisable in light of high sensitivity rate of Gram negative bacteria involved in wound infection. Since the local sensitivity data has prominent role in development of guidelines for treatment of wound infection empirically, similar large scale studies would help in developing such local guidelines minimising unnecessary use of broad spectrum antibacterial agents and decreasing the development of antimicrobial resistance.

\section{ACKNOWLEDGEMENT}

To the microbiology laboratory technologists and other staffs of Department of Microbiology, B and B Hospital Gwarko, Lalitpur.

To cite this article: Suwal SN, Kaundinnyayana S, Prajapati KG, Baidya R. Sensitivity pattern of gram negative bacteria contaminating traumatic wounds: A retrospective study in a tertiary care hospital of Kathmandu Valley, Nepal. MJSBH. 2020;19(2):103-7.

Conflict of Interest: None declared

\section{REFERENCES}

1. Robson MC. Wound infection: A failure of wound healing caused by an imbalance of bacteria. Surg Clin North Am. 1997;77:637. DOI: 10.1016/s0039-6109(05)70572-7

2. Beilman GJ, Dunn DL. Surgical site infections. In: Brunicardi FC.editor. Schwartz's Principles of Surgery. 10th edition. McGraw-Hill Education; 2015. p135-59.

3. Parajuli NP, Acharya SP, Mishra SK, Parajuli K, Rijal BP, Pokhrel BM. High burden of antimicrobial resistance among gram negative bacteria causing healthcare associated infections in a critical care unit of Nepal. Antimicrob Resist Infect Control. 2017;6:67. DOI: 10.1186/s13756-017-0222-z

4. Rijal BP, Satyal D, Parajuli NP. High Burden of Antimicrobial Resistance among Bacteria Causing Pyogenic Wound Infections at a Tertiary Care Hospital in Kathmandu, Nepal. J Pathog. 2017;2017:9458218. DOI: https:// doi.org/10.1155/2017/9458218.

5. Malik KI, Malik MA, Aslam A. Honey compared with silver sulfadiazine in the treatment of superficial partial thickness burns. Int Wound Journal. 2010;7:413-170. DOI: 10.1111/j.1742-481X.2010.00717.X.

6. Polk RE, Fishman NO. Antimicrobial Stewardship. In: Madell GL, Bennet JE, Dolin R. editors. Mandell, Doughlas and Bennets Principles and Practice of Infectious Diseases. 7th edition. Churchil Livingstone, Elsevier, Philadelphia; 2010. p 682. 
7. Clinical and Laboratory Standards Institute. CLSI document M100-S23. Performance standards for antimicrobial susceptibility testing; Twenty third informational supplement Edition. CLSI; 2013.

8. Li B, Ke B, Zhao X, Guo Y, Wang W, Wang X, et al. Antimicrobial Resistance Profile of $m c r-1$ Positive Clinical Isolates of Escherichia coli in China From 2013 to 2016. Front Microbiol. 2018;9:2514. DOI: 10.3389/fmicb. 2018.02514.

9. KC R, Shrestha A, SharmaV. Bacteriological Study of Wound Infection and Antibiotic Susceptibility Pattern of the Isolates. NJST. 2014;14(2):143-50. DOI:10.3126/njst.v14i2.10428.

10. Rai S, Yadav UN, Pant ND, Yakha JK, Tripathi PP, Poudel A, et al. Bacteriological Profile and Antimicrobial Susceptibility Patterns of Bacteria Isolated from Pus/Wound Swab Samples from Children Attending a Tertiary Care Hospital in Kathmandu, Nepal. Int J Microbiol. 2017;2017:2529085. DOI: 10.1155/2017/2529085.

11. Upreti N, Rayamajhee B, Sherchan SP, Choudhari MK, Banjara MR. Prevalence of methicillin resistant Staphylococcus aureus, multidrug resistant and extended spectrum $\beta$-lactamase producing gram negative bacilli causing wound infections at a tertiary care hospital of Nepal. Antimicrob Resist Infect Control. 2018;7:121. DOI: 10.1186/s13756-018-0408-z

12. Mythri BA, Patil AB, Arati K, Sharon VA. Aerobic Bacteriological Profile from Wound Site Infections in Road Traffic Accident (RTA) Patients. Indian J Microbiol Res. 2016;3(1):37-39. DOI:10.5958/2394-5478.2016.00010.8

13. Lim LM, Ly N, Anderson D, Yang JC, Macander L, Jarkowski A, et al. Resurgence of colistin: a review of resistance, toxicity, pharmacodynamics and dosing. Pharmacotherapy. 2010;30:1279-91. DOI: 10.1592/phco. 30.12 .1279

14. Karabinis A, Paramythiotou E, Mylona-Petropoulou D, Kalogeromitros A, Katsarelis N, Kontopidou F, et al. Colistin for Klebsiella pneumoniae - Associated Sepsis. Clinical Infectious Diseases. 2004;38(1):7-9. DOI: https:// doi.org/10.1086/380461.

15. Rojas LJ, Salim M, Cober E, Richter SS, Perez F, Salata RA, et al. for the Antibacterial Resistance Leadership Group. Colistin Resistance in Carbapenem-Resistant Klebsiella pneumoniae: Laboratory Detection and Impact on Mortality. Clin Infect Dis. 2017;64(6):711-18. DOI: https://doi.org/10.1093/cid/ciw805

16. Dickstein Y, Lellouche J, Schwartz D, Nutman A, Rakovitsky N, Benattar YD, et al. Colistin Resistance Development Following Colistin-Meropenem Combination Therapy vs. Colistin Monotherapy in Patients with Infections Caused by Carbapenem-Resistant Organisms. Clinical Infectious Diseases. 2019;1146:1-9. DOI:https:// doi.org/10.1093/cid/ciz1146

17. Livermore DM. Current epidemiology and growing resistance of gram-negative pathogens. Korean J Intern Med . 2012;27(2):128-42. DOI: 10.3904/kjim.2012.27.2.128 